\title{
Increased mean platelet volume in septicaemia
}

\author{
J VAN DER LELIE, AEG Kr VON DEM BORNE \\ From the Department of Medicine and Department of Haematology, Academic Medical Centre, University of \\ Amsterdam, Amsterdam, The Netherlands
}

SUMMARY An increased mean platelet volume (MPV), measured by the Coulter counter model S plus, was found in 13 of 25 patients with proven septicaemia but in none of 25 patients with localised bacterial infection and negative blood cultures. The increase in MPV was found both in patients with normal and low platelet counts and was not related to a particular micro-organism. Patients who responded favourably to antibiotic treatment all had normal MPVs after one week of treatment. However, 9 of 11 patients with a prolonged course of their infection due to endocarditis or abdominal abscesses had raised MPVs after seven days of treatment, and four patients who died of infection in the first week all had increased MPVs on the day of their death. An increased MPV in a patient with bacterial infection possibly indicates that the infection has become invasive - that is, that septicaemia has occurred. A persistent rise or further increase indicates that treatment is inadequate.

Until recently platelet size determination was too elaborate to be used in routine daily practice. The introduction of the new electronic cell counters, such as the Coulter counter model S plus, however, provides a mean platelet volume (MPV), comparable to the MCV of erythrocytes, on each whole blood sample that is processed. ${ }^{12}$ This makes it possible to study platelet size in a great variety of clinical conditions.

MPV can be useful to differentiate between thrombocytopenia of central and peripheral origin ${ }^{3}$ and to predict haemorrhagic diathesis in thrombocytopenic patients. ${ }^{4}$

The usefulness of MPV in patients with normal platelet counts has not yet been established. We report increases of MPV in patients with septicaemia both in low and in normal platelet count range. Possible mechanisms and clinical relevance are discussed.

\section{Patients and methods}

\section{PATIENTS}

We studied two different types of patients: group I consisted of 25 patients from the medical and the surgical department, who all had both the clinical signs and symptoms of septicaemia and one or more positive blood cultures. Patients with a haematological disease and patients treated with cytostatic drugs were not included in the study. Diagnoses were: cholecystitis and cholangitis in 6, peritonitis in 5 , endocarditis in 5, urosepsis in 4, penumonia in 2 , erysipelas in 2 , and osteomyelitis in 1 patient. Twelve patients had Gram-negative sepsis, 11 Gram-positive sepsis and two patients had combined Gram-negative and -positive sepsis. Microorganisms cultured were: $E$ coli in 11, B fragilis in 3, $S$ marcescens in $1, P s$ aeruginosa in $1, H$ influenzae in $1, S$ aureus in 6 and streptococci in 7 patients.

Group II consisted also of 25 patients with documented bacterial infections, but these patients had no clinical signs of septicaemia and blood cultures remained negative. Diagnoses in these patients were: airway infection in 15 , urinary tract infection in 4 , gastroenteritis in 4 , and skin infections in two patients.

\section{BLOOD CULTURES}

For culture $10 \mathrm{ml}$ of blood, collected under sterile conditions, was distributed in equal parts over two bottles. One contained $95 \mathrm{ml}$ of Columbia broth with $0.05 \%$ Liquoid and was vented on arrival at the laboratory for aerobic culture. The other one contained $95 \mathrm{ml}$ of Brewer's thioglycollate with $0.05 \%$ Liquoid for anaerobic culture and was not vented. All bottles were subcultured aerobically and anaerobically after seven days of incubation or earlier if growth was suspected at inspection. 
vacuum tubes containing liquid $K_{3}$ EDTA as an anticoagulant (Venoject, Terumo, Belgium). The samples were analysed by the Coulter counter model S plus (Coulter Electronics, Hialeah, Florida) in our laboratory within six hours after collection. Quality control and calibration of the Coulter counter was performed according to the instructions of the manufacturer. The normal value for the MPV in our laboratory is $7 \cdot 3 \pm 1.4 \mathrm{fl}$ (mean $\pm 2 \mathrm{SD})$. The mean intraindividual variation for the MPV, measured three times with monthly intervals in 10 normal persons, was $0.4 \mathrm{fl}$ (range 0.0-0.9 fl). Prothrombin time and activated partial thromboplastin time, for overall blood coagulation screening, were measured according to standard procedures. The ethanol gelation test, to detect circulating fibrin monomers as an indirect measure of intravascular thrombin generation, was performed as described by Godal and Abildgaard. 5

\section{Results}

An increased MPV on the day the first blood culture was positive, was found in 13 of the 25 patients with septicaemia, both in patients with normal and in those with low platelet counts (Fig. 1) Six patients had a platelet count below $100 \times 10^{\circ} / 1$. Mean platelet number in the group of patients with increased MPV was lower and prothrombin time and partial thromboplastin time were longer than in the group with normal MPV (Table 1). However, the difference was not statistically significant. The 25 patients

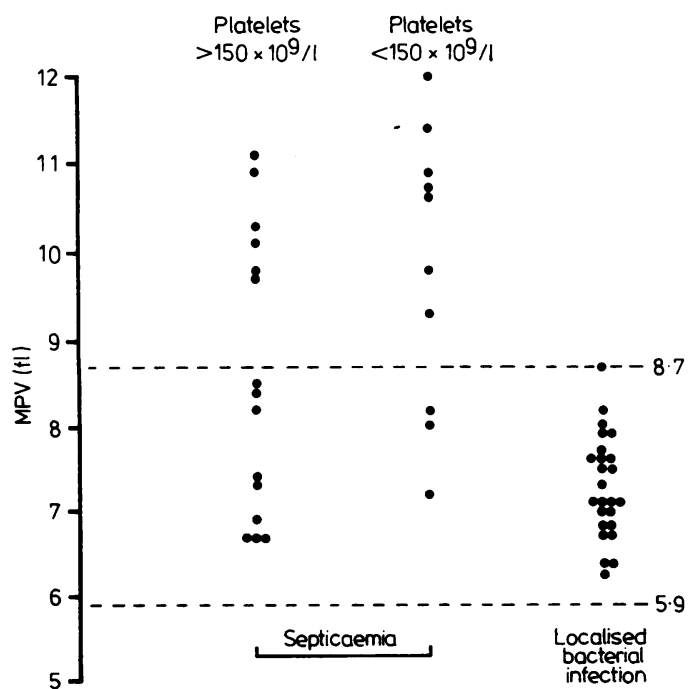

Fig. 1 MPV on the day of the first positive blood culture in patients with septicaemia and MPV in patients with localised bacterial infection.
Table 1 Platelet count, prothrombin time and partial thromboplastin time (mean $\pm S D$ ) in 25 patients with septicaemia on the day of the first positive blood culture. Differences not significant (Wilcoxon rank test).

\begin{tabular}{lcc}
\hline & $\begin{array}{l}\text { Normal MPV at } \\
\text { diagnosis }(n=12)\end{array}$ & $\begin{array}{c}\text { MPV increased at } \\
\text { diagnosis }(n=13)\end{array}$ \\
\hline Platelet count $\left(\times 10^{9} / 1\right)$ & $231 \pm 93$ & $165 \pm 108$ \\
$\begin{array}{l}\text { Prothrombin time }(\mathrm{s}) \\
\text { Partial thromboplastin }\end{array}$ & $12.9 \pm 0.7$ & $13.8 \pm 1.4$ \\
$\quad$ & $48 \pm 10$ & $55 \pm 10$ \\
\hline
\end{tabular}

with localised bacterial infections and negative blood cultures all had MPV's within the normal range (Fig. 1). They all had platelet counts above $100 \times 10^{9} / 1$. A rise of the MPV in the patients with sepsis was not related to particular micro-organisms. MPV was raised in 6 of 11 patients with Gram-positive sepsis, in 5 of 12 patients with Gramnegative sepsis, and in both patients in whom Gram-negative and -positive micro-organisms were cultured. The ethanol gelation test, as an indicator for disseminated intravascular coagulation, was positive in one patient with sepsis and normal MPV and in two patients with sepsis and increased MPV.

At light microscopic examination of JennerGiemsa stained peripheral blood films, in patients with increased MPV, swollen and partially degranulated platelets were often seen.

Ten patients in the septicaemia group responded quickly to antibiotic treatment. All of them had a normal MPV after one week of treatment (Table 2). Fifteen patients with sepsis had a prolonged course of their infection with persistently high temperature and in five of them repeatedly positive blood cultures. This group includes the five patients with endocarditis. eight patients with abdominal infections and abscesses (three of whom died in the first week of treatment) and two patients with fulminant pneumonia (one of whom died after four days). Only two patients in this group had a normal MPV after one week of treatment (Table 2). The four

Table 2 Platelet count and MPV (mean $\pm S D$ ) in patients with septicaemia on the day of the first positive blood culture and after seven days of treatment.

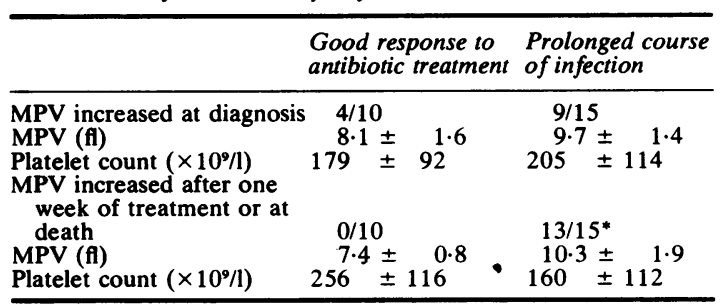

*Four patients died within the first week. 


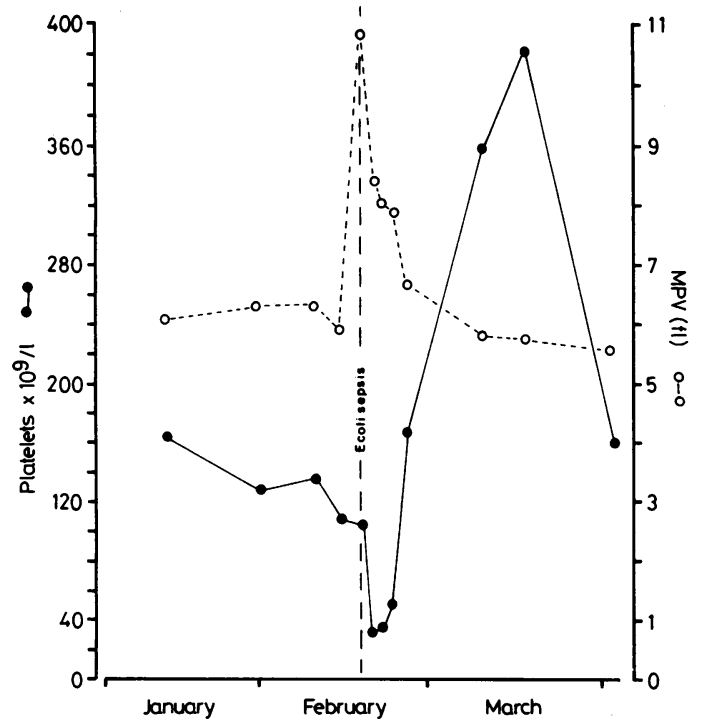

Fig. $2 M P V$ and platelet count in a patient with $E$ coli sepsis after retrograde IVP (see text).

patients who died in the first week all had an increased MPV on the day of their death. In 10 patients MPVs were available from the days before the onset of septicaemia. This was in the normal range in all of them except one. This patient suffered from a large inguinal haematoma, after cardiac catheterisation, and developed erysipelas in the region of this haematoma and streptococci sepsis. On the day the first blood culture was positive, MPV was raised in four of these 10 patients.

It appeared that the MPV increase at the beginning of illness may occur very rapidly. An illustrative case is that of a man with a malfunctioning kidney transplant. His urinary tract was chronically infected with $E$ coli. A retrograde IVP of the transplant kidney was performed to exclude anatomical obstruction. A few hours later the patient developed chills, high temperature and low blood pressure. E coli was isolated from his blood. There was a direct increase in MPV, followed by a drop in platelet count. The transplant kidney was removed and MPV and platelet count returned to normal (Fig. 2).

\section{Discussion}

Septicaemia frequently involves platelets and can cause severe thrombocytopenia. ${ }^{6}$ Platelet size in bacterial infection thus far has received little attention. Nelson and $\mathrm{Kehl}^{3}$ reported an increased MPV, measured by Coulter counter model $S$ plus, in patients with thrombocytopenia due to platelet loss or consumption, including a small number of patients with acute infection. Giles, ${ }^{7}$ also using a Coulter counter model S plus, found in patients with infection an increased MPV in only $3.6 \%$ of the patients. However, he did not differentiate between localised infection and septicaemia. The MPV in patients with localised infection in our study was normal in all, but about half of the patients with septicaemia had increased MPV, not related to the platelet count. Moreover, a further increase was noticed if treatment was unsatisfactory, and a normalisation in patients treated adequately. Thus an increased MPV in a patient with bacterial infection could suggest the possibility that the infection has become invasive-that is, that septicaemia has occurred. A persistent rise or a further increase might indicate that treatment is inadequate.

Large platelets are thought by some ${ }^{8}$ to be young platelets, recently released from the bone marrow, which become smaller with age. However, others ${ }^{910}$ postulate that platelet size is not related to age, but to the ploidy class of the megakaryocyte which produces the platelets. Increase of megakaryocyte ploidy occurs in cases of enhanced thrombocytopoiesis. Therefore, it is possible that the rise in MPV in septicaemia is caused by an increased production of larger and/or younger platelets as a reaction to septicaemia-related platelet destruction. The raised MPV in patients with normal platelet counts should then reflect a compensated thrombocytolytic state. ${ }^{11}$ However, the presence of an increased MPV at diagnosis and the remarkable rise of MPV, which can be found at the beginning of septicaemia (Fig. 2), make that the production of larger platelets cannot be the only cause for the MPV increase in septicaemia. A sudden onset rather suggests an external influence on the circulating platelets.

In patients with acute bacterial infection increased levels of $\beta$-thromboglobulin have been found, indicating in vivo activation and release of platelets. ${ }^{12}$ We therefore postulate that in vivo activation and release can be the cause for the direct MPV increase in septicaemia, presuming that activated and released platelets, which are less dense, are also larger. The finding in light microscopy of large degranulated platelets in patients with septicaemia and increased MPV could well fit in in this hypothesis.

There are several possible causes for platelet activation and release in septicaemia. In vitro platelet serotonin release has been demonstrated when platelets were incubated with endotoxin ${ }^{13}$ and pneumococci. ${ }^{14}$ Also activated complement ${ }^{15}$ and thrombin ${ }^{16}$ can cause platelet release reaction. Both complement activation and thrombinaemia can be found in septicaemia. ${ }^{17} 18$ Moreover, platelets acti- 
vated and released by thrombin can remain in the circulation. ${ }^{16}$ In our study only three of the 25 patients with sepsis had unequivocally intravascular thrombin generation, indicated by a positive ethanol gelation test, one had a normal and two had an increased MPV. However, patients with sepsis and increased MPV had slightly, but statistically nonsignificant, longer prothrombin and partial thromboplastin times than patients with sepsis and normal MPV (Table 1). This might indicate a smaller degree of intravascular thrombin formation in the patients with increased MPV.

The authors acknowledge Dr PJGM Rietra for his microbiological support and Nick van de IJssel for his technical assistance.

\section{References}

'Besman JD. Evaluation of automated whole blood platelet counts and particle sizing. Am J Clin Pathol 1980;74:157-62.

${ }^{2}$ Rowan RM, Frazer C, Gray JH, McDonald GA. The Coulter counter model S plus-The shape of things to come. Clin Lab Hematol 1979;1:29-40.

${ }^{3}$ Nelson RB, Kehl D. Electronically determined platelet indices in thrombocytopenic patients. Cancer 1981;48:954-6.

${ }^{4}$ Eldor A, Avitzour M, Or R, Hanna R, Penchas S. Prediction of haemorrhagic diathesis in thrombocytopenia by mean platelet volume. Br Med J 1982;285:397-400.

${ }^{5}$ Godal MC, Abildgaard U. Gelation of soluble fibrin in plasma by ethanol. Scand J Haematol 1966;3:342-50.

- Corrigan JJ. Thrombocytopenia: A laboratory sign of septicaemia in infants and children. J Pediatr 1974;85:219-21.
' Giles C. The platelet count and mean platelet volume. Br J Haematol 1981;48:31-7.

${ }^{8}$ Karpatkin S. Heterogeneity of human platelets. I. Metabolic and kinetic evidence suggestive of young and old platelets. J Clin Invest 1969;48:1073-82.

${ }^{9}$ Paulus JM. Platelet size in man. Blood 1975;46:321-36.

${ }^{10}$ Pennington DG, Lee NLY, Roxburgh AE, McGready JR. Platelet density and size: The interpretation of heterogeneity. Br J Haematol 1976;34:365-76.

"Karpatkin S, Garg SK, Siskind GW. Auto-immune thrombocytopenic purpura and the compensated thrombocytolytic state. Am J Med 1971;51:1-4.

${ }_{12}$ Douglas JT, Lowe GDO, Forbes CD, Prentice CRM. $\beta$-thromboglobulin and platelet counts-Effect of malignancy, infection, age and obesity. Thromb Res 1982;25:495-64.

${ }^{13}$ Hawiger J, Hawiger A, Steckley S, Timmons S, Cheng C. Membrane changes in human platelets induced by lipopolysaccharide endotoxin. Br J Haematol 1977;35:285-99.

${ }^{14}$ Zimmerman TS, Spiegelberg HL. Pneumococcus-induced serotonin release from human platelets. Identification of the participating plasma-serum factor as immunoglobulin. $J$ Clin Invest 1975;56:828-34.

is Meuer S, Echer U, Hadding U, Bitter-Suermann D. Platelet serotonin release by $\mathrm{C} 3 \mathrm{a}$ and $\mathrm{C} 5 \mathrm{a}$ : Two independent pathways of activation. J Immunol 1981;126:1506-09.

${ }^{16}$ Reimers HJ, Kinlough-Rathbone RL, Carenave JP, et al. In-vitro and in-vivo functions of thrombin treated platelets. Thromb Haemost 1976;35:151-66.

${ }^{17}$ McCabe WR. Serum complement levels in bacteraemia due to Gram-negative organisms. N Engl J Med 1973;288:21-3.

${ }^{18}$ Corrigan JJ, Ray WL, May N. Changes in the blood coagulation system associated with septicaemia. $N$ Engl J Med 1968;279:851-6.

Request for reprints to: Dr J van der Lelie, Department of Haematology, Academic Medical Centre, Meibergdreef 9, $1105 \mathrm{AZ}$ Amsterdam, The Netherlands 